\title{
Commissioning and initial experimental program of the BGO-OD experiment at ELSA
}

S. Alef ${ }^{1}$, P. Bauer ${ }^{1}$, D. Bayadilov ${ }^{2}$, R. Beck ${ }^{2}$, M. Becker ${ }^{2}$, A. Bella ${ }^{1}$, P. Bielefeldt ${ }^{1}$, S. Böse ${ }^{2}$, A. Braghieri, ${ }^{3}$, K. Brinkmann ${ }^{4}$, P. Cole ${ }^{1,5}$, R. Di Salvo ${ }^{6}$, H. Dutz ${ }^{1}$, D. Elsner ${ }^{1}$, A. Fantini ${ }^{6,7}$, O. Freyermuth ${ }^{1}$, S. Friedrich ${ }^{1}$, F. Frommberger ${ }^{1}$, V. Ganenko ${ }^{8}$, D. Geffers ${ }^{1}$, G. Gervino ${ }^{9,10}$, F. Ghio ${ }^{11,12}$, S. Görtz ${ }^{1}$, A. Gridnev ${ }^{13}$, E. Gutz ${ }^{4}$, D. Hammann ${ }^{1}$, J. Hannappel ${ }^{1}$, W. Hillert ${ }^{1}$, A. Ignatov ${ }^{14}$, R. Jahn ${ }^{2}$, R. Joosten ${ }^{2}$, T.C. Jude ${ }^{1}$, F. Klein ${ }^{1}$, J. Knaust ${ }^{1}$, K. Kohl ${ }^{1}$, K. Koop ${ }^{2}$, B. Krusche ${ }^{15}$, A. Lapik ${ }^{14}$, P. Levi Sandri ${ }^{16, \star}$, I.V. Lopatin ${ }^{13}$, G. Mandaglio ${ }^{17,18}$, F. Messi ${ }^{1}$, R. Messi ${ }^{6,7}$, V. Metag ${ }^{4}$, D. Moricciani ${ }^{6}$, A. Mushkarenkov ${ }^{14}$, M. Nanova ${ }^{4}$, V. Nedorezov ${ }^{14}$, D. Novinskiy ${ }^{13}$, P. Pedroni ${ }^{3}$, B. Reitz ${ }^{1}$, M. Romaniuk ${ }^{6}$, T. Rostomyan ${ }^{1}$, N. Rudnev ${ }^{14}$, C. Schaerf ${ }^{6,7}$, G. Scheluchin ${ }^{1}$, H. Schmieden ${ }^{1}$, A. Stugelev ${ }^{13}$, V. Sumachev ${ }^{13}$, V.Tarakanov ${ }^{13}$, V. Vegna ${ }^{1}$, D. Walther ${ }^{1}$, D. Watts ${ }^{19}$, H. Zaunick ${ }^{2}{ }^{4}$, and T. Zimmermann ${ }^{1}$

${ }^{1}$ Physikalishes Institut, Nußallee 12, 53115, Bonn, Germany

${ }^{2}$ Helmoltz-Institut für Strahlen- und Kernphysik, Nußallee 14-16, 53115 Bonn, Germany

${ }^{3}$ INFN sezione di Pavia, Via Agostino Bassi, 6 - 27100 Pavia, Italy

${ }^{4}$ Justus-Liebig-Universität, II. Physikalisches Institut, Heinrich-Buff-Ring 16, 35392 Gießen, Germany

${ }^{5}$ Idaho State University, 921 S. 8th Ave., Pocatello, Idaho 83209, USA

${ }^{6}$ INFN Roma Tor Vergata, Via della Ricerca Scientifica 1, 00133 Roma, Italy

${ }^{7}$ Universitá di Roma Tor Vergata, Via della Ricerca Scientifica 1, 00133 Roma, Italy

${ }^{8}$ Kharkov Institute of Physics and Technology, Akademicheskaya St. 1, Kharkov, 61108, Ukraine

${ }^{9}$ INFN sezione di Torino, Via P.Giuria 1, 10125 Torino, Italy

${ }^{10}$ Universitá di Torino, via P. Giuria 1, 10125 Torino, Italy

${ }^{11}$ INFN sezione di Roma, P.le Aldo Moro, 2, 00185 Roma, Italy

${ }^{12}$ Istituto Superiore di Sanitá, Viale Regina Elena 299, 00161 Roma, Italy

${ }^{13}$ Petersburg Nuclear Physics Institute, Gatchina, Leningrad District, 188300, Russia

${ }^{14}$ Russian Academy of Sciences Institute for Nuclear Research, prospekt 60-letiya Oktyabrya 7a, Moscow 117312, Russia

${ }^{15}$ Institut für Physik, Klingelbergstraße 82, 4056 Basel, Switzerland

${ }^{16}$ INFN Laboratori Nazionali di Frascati, Via E. Fermi 40, 00044 Frascati, Italy

${ }^{17}$ INFN sezione Catania, 95129 Catania, Italy

${ }^{18}$ Universitá degli Studi di Messina, Via Consolato del Mare 41, 98121 Messina, Italy

${ }^{19}$ The University of Edinburgh, James Clerk Maxwell Building, Mayfield Road, Edinburgh EH9 3JZ, UK

\begin{abstract}
BGO-OD is a new meson photoproduction experiment at the ELSA facility of Bonn University. It aims at the investigation of non strange and strange baryon excitations, and is especially designed to be able to detect weekly bound meson-baryon type structures. The setup for the BGO-OD experiment is presented, the characteristics of the photon beam and the detector performances are shown and the initial experimental program is discussed.
\end{abstract}

^e-mail: Paolo.Levisandri@lnf.infn.it 


\section{Introduction}

The BGO-OD Collaboration was formed at the end of 2010 with the aim of studying meson photoproduction off the nucleon (and eventually off light nuclei) at Bonn ELSA. The photon beam covers the region between 0.3 to $2.9 \mathrm{GeV}$, and substantial linear polarization can be obtained up to $1.8 \mathrm{GeV}$ via coherent Bremsstrahlung. The detector covers $\sim 95 \%$ of $4 \pi$ and is optimized for the measurement of final states with both charged and neutral particles. We present here the beam characteristics, the detector performances, as obtained in the commissioning runs of the experiment, and we discuss the main scientific goals and the initial experimental program.

\section{The photon beam}

The $\gamma$-ray beam for BGO-OD is obtained through Bremsstrahlung of the $3.2 \mathrm{GeV}$ electrons extracted from the ELSA stretcher ring [1]. Amorphous copper radiators of different thicknesses are available and a diamond radiator can be inserted as well, allowing production of a linearly polarized photon beam. The energy of the photons is determined by the tagging technique: the electrons that have emitted Bremsstrahlung photons are momentum-analyzed by a tagger magnet and their position is measured by a tagger counter formed by 120 scintillators ( 160 ps time resolution, $0.5 \%-2.0 \%$ energy resolution, and capable of handling up to $50 \mathrm{MHz}$ event rate). The tagger is part of the event trigger. In order to have a more precise measurement of the photon energy (and thus of its polarization) under the coherent peak, the tagger is complemented with the ARGUS hodoscope formed by 480 scintillating fibers. The energy resolution of the ARGUS is $\sim 0.08 \%$. In Fig. 1 we show the relative rate (diamond/copper) as measured by the tagger and by $A R G U S$.

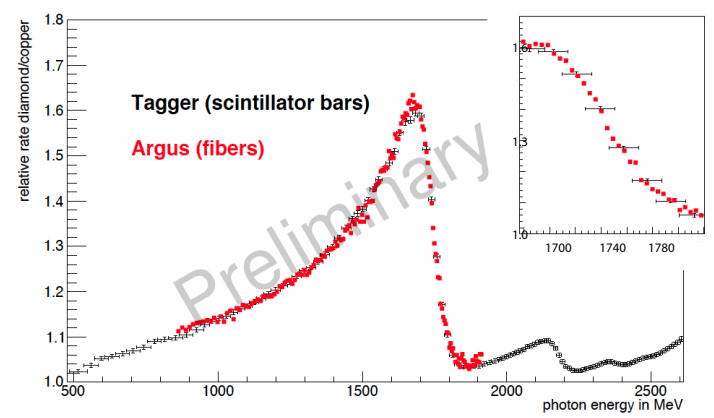

Figure 1. Relative count rate (diamond/copper) as measured by the tagger (scintillator bars, black points), and by the ARGUS hodoscope (scintillating fibers, red squares). In the insert, the detail of the coherent edge region.

\section{The detector}

The BGO-OD detector is formed by coupling the Rugby Ball calorimeter previously used at the GrAAL experiment with the Open Dipole magnetic spectrometer. The detector, shown in Fig. 2, can be divided into three parts according to the different polar angle coverage. The central part $\left(155^{\circ} \leq \theta \leq 25^{\circ}\right)$ is covered by the Rugby Ball BGO calorimeter, formed by 480 crystals with PMT readout and equipped with sampling ADCs. The target (liquid H2 or D2 or solid) sits in the center of the calorimeter and is surrounded by ancillary detectors (cylindrical MWPC and scintillator Barrel) 
for particle tracking and identification. The polar angles between $8^{\circ}$ and $25^{\circ}$ are covered by a ring of scintillating counters (SciRi) and by MRPC chambers (Daisy) with excellent time resolution. Finally, the forward region is covered by the Open Dipole forward spectrometer. Its central part is a $0.45 \mathrm{~T}$ large aperture dipole magnet (provided by DESY on permanent loan basis). Tracking is performed by position detectors in front of the magnet (MoMo and Scifi2), both consisting of scintillating fibers, and 8 double layer Drift Chambers followed by 3 ToF scintillating walls behind it.

\section{BGO-OD setup}

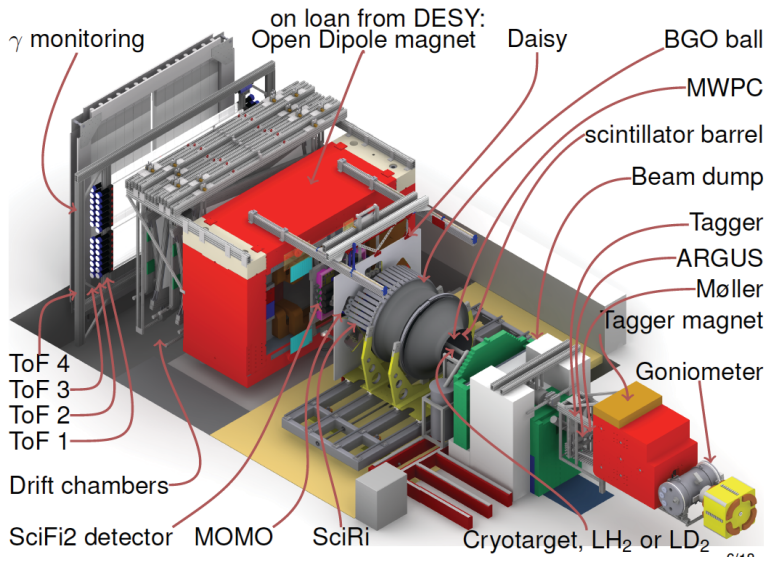

Figure 2. Schematic view of the BGO-OD experimental setup. Electron beam enters from the bottom-right corner.

The performance of the BGO Rugby Ball is summarized in Fig. 3 where we show the two-photon invariant mass spectrum from a proton target obtained with kinematical fit and confidence cut. The $\pi^{0}, \eta, \omega$ and $\eta^{\prime}$ peaks are all clearly visible. The energy calibration is obtained using a Na-22 source with peak at $1.275 \mathrm{MeV}$.

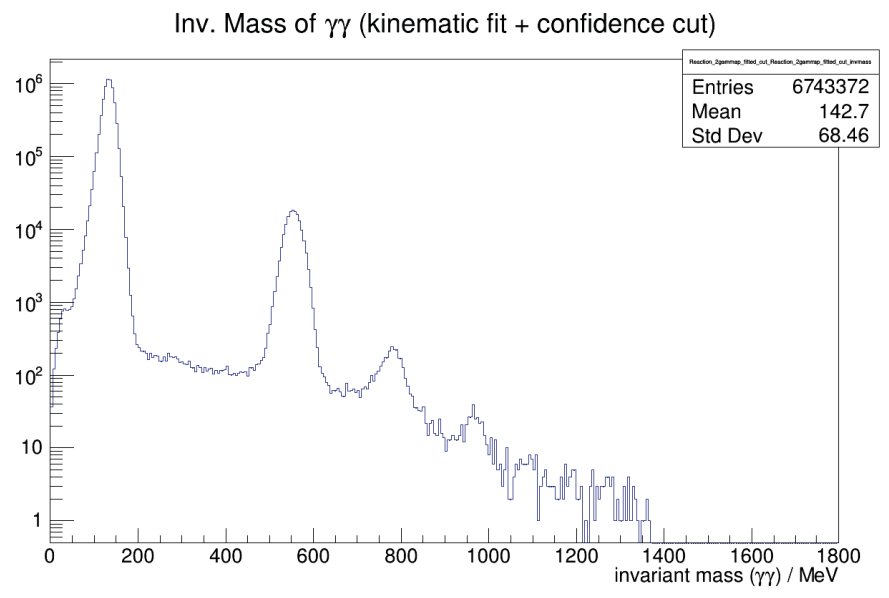

Figure 3. The two-photons invariant mass from a proton target measured by the Rugby Ball calorimeter.

The Rugby Ball can also measure protons[2] and neutrons[3], and by using sampling ADCs with $\sim 2$ ns time resolution it can also measure $K^{+}$following a method originally developed in Ref.[4]. Positive kaons are stopped within the crystals of the Rugby Ball up to a kinetic energy of approximately 
$400 \mathrm{MeV}$. The time resolution allows to distinguish the signal of the stopped $K^{+}$from the signal of the subsequent weak decay $K^{+} \rightarrow \mu^{+} v$. (see Fig. 4).
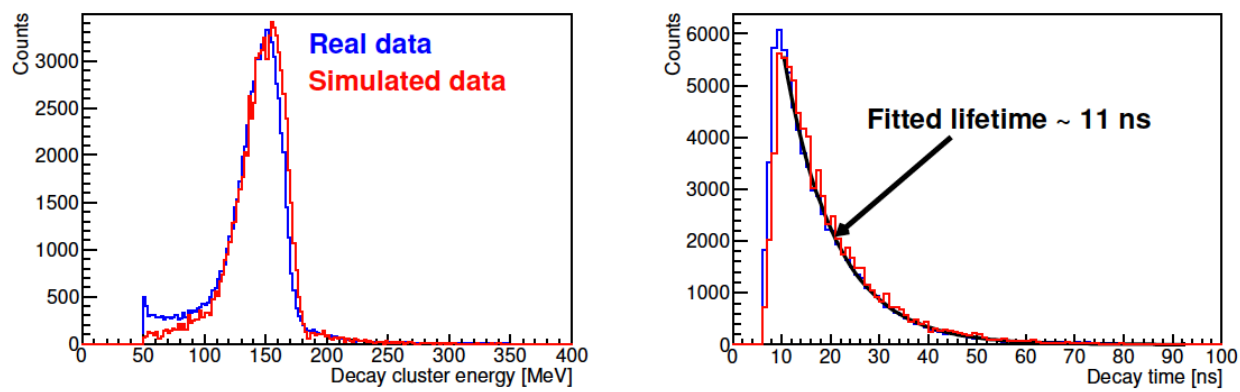

Figure 4. Left: energy of the delayed signal from the decay $K^{+} \rightarrow \mu^{+} v$. The peak at $153 \mathrm{MeV}$ corresponds to the $\mu^{+}$energy deposition from a $K^{+}$decay at rest. Right: reconstructed $K^{+}$lifetime.

In the forward region, particle identification is performed by looking at the bidimensional plot of particle velocity versus particle momentum (Fig. 5). By selecting protons or charged kaons in this plot we can obtain the two missing mass plots shown in Fig. 6.
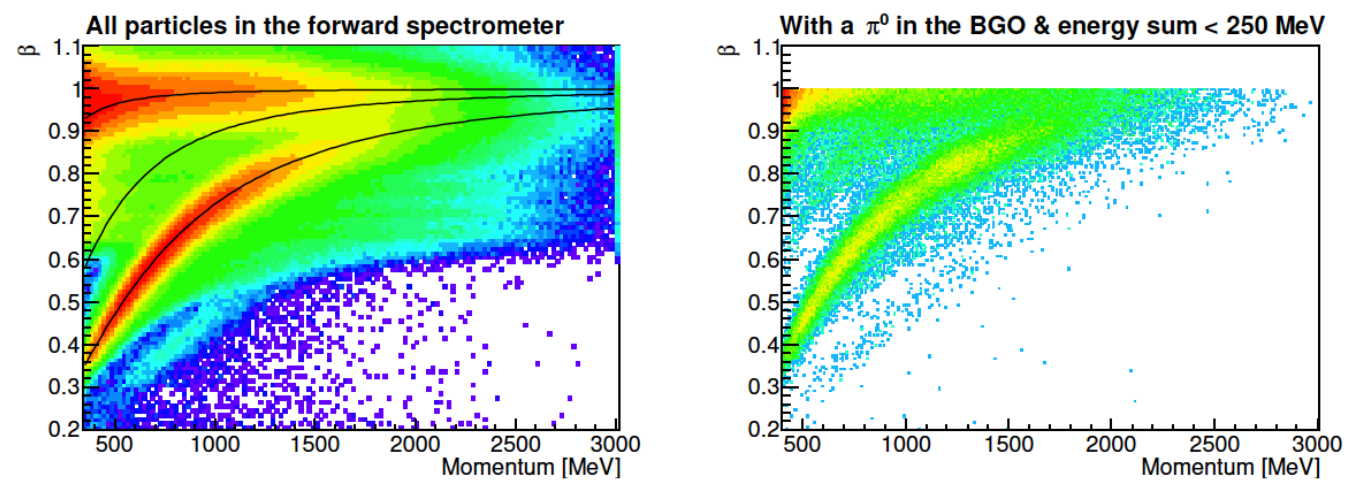

Figure 5. Left: $\beta$ vs. momentum for all particles detected in the Open Dipole forward spectrometer. Right: same plot in coincidence with a $\pi^{0}$ in the calorimeter and with energy sum in the calorimeter less than $250 \mathrm{MeV}$. The $K^{+}$line is accentuated and is clearly visible.

\section{Experimental program}

The setup is ideal for the investigation of reactions dominated by $\mathrm{t}$-channel mechanisms due to the acceptance at forward angles with high momentum resolution. The unique combination of excellent calorimetry coupled with high resolution forward tracking is well suited to investigate reaction channels with open strangeness and complicated final states. Strangeness photoproduction off the proton (with final states $K^{+} \Lambda, K^{+} \Sigma^{0}, K^{0} \Sigma^{+} \ldots$ ) will be accessed, and neutral particle identification in the central region helps to determine final states of mixed charge. The cusp-like structure observed by the 

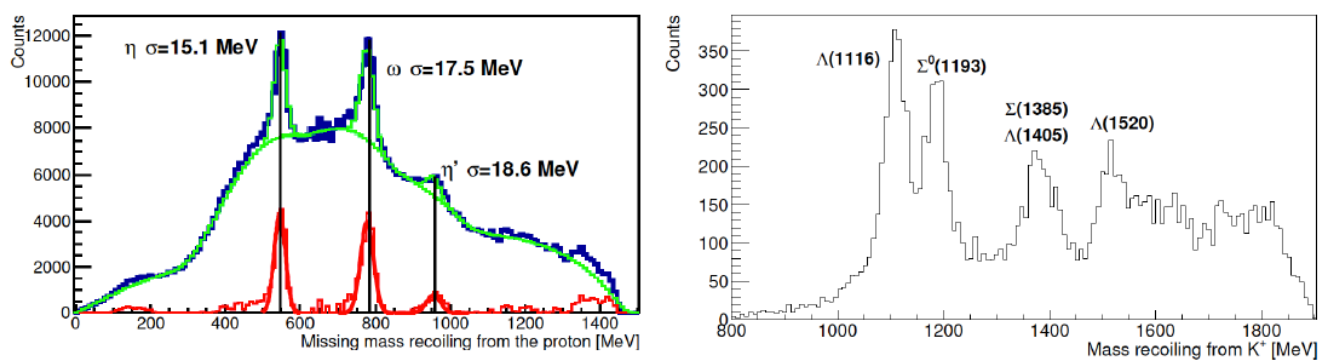

Figure 6. Missing mass spectrum in the Open Dipole forward spectrometer. Left: from the proton. $\eta, \omega$ and also $\eta^{\prime}$ peaks are clearly visible. Right: from the $K^{+} . \Lambda$ and $\Sigma^{0}$ peaks are very clear and also other hyperons peaks are present.

CBELSA/TAPS collaboration[5] will be investigated by measuring the differential cross section in $\gamma p \rightarrow K^{0} \Sigma^{+}$and the beam asymmetry over the $K^{*}$ threshold region, achieving high statistics by including data of both neutral and charged decays of the $K^{0}$ and $\Sigma^{+}$. The acceptance of the Open Dipole forward spectrometer is also ideal to measure data at centre-of-mass angles $\theta_{c m} \leq 15^{\circ}$ for $K^{+} Y$, where data are scarce and incompatible. A central goal is to disentangle the structure of hyperon excitations, e.g. the $\Lambda(1405)$ or hypothesised $\Sigma^{*}$ states of $J^{P}=1 / 2^{-}$in the same mass range.

Pseudoscalar and vector meson photoproduction will be studied too, both off the proton and off the neutron, measuring cross sections and beam asymmetries. Thanks to the high energy resolution for the incoming photon energy provided by the ARGUS hodoscope, the beam asymmetry in $\eta^{\prime}$ photoproduction will be measured at threshold, allowing for a more detailed study of the strong energy dependence observed by the GrAAL collaboration[6], and at higher energy (up to $1800 \mathrm{MeV}$ ) where no experimental data are available yet. The high efficiency of the Rugby Ball for neutron detection will allow the measurements of $\eta$ and $\omega$ off the quasi-free neutron with liquid D2 target. Finally, the $\Phi$ photoproducion will be investigated as well.

\section{Conclusion}

The BGO-OD experiment is commissioned; the performances of the beam and of the apparatus are as expected, production runs are underway and data analysis is ongoing for many reaction channels. The experimental program includes meson photoproduction from proton, deuteron and light nuclei.

\section{References}

[1] W. Hillert, Eur. Phys. J. A 28, 139 (2006)

[2] A. Zucchiatti et al., Nucl. Instr. and Meth. A 321, 219 (1992)

[3] O. Bartalini et al., Nucl. Instr. and Meth. A 562, 85 (2006)

[4] T.C. Jude, D.I. Glazier and D.P. Watts, Phys. Lett. B 735, 112 (2014)

[5] R. Ewald et al., Phys. Lett. B 713, 180 (2014)

[6] P. Levi Sandri et al., Eur. Phys. J. A 51, 77 (2015) 